University of Nebraska - Lincoln

DigitalCommons@University of Nebraska - Lincoln

2006

\title{
Immunogenicity of combination DNA vaccines for Rift Valley fever virus, tick-borne encephalitis virus, Hantaan virus, and Crimean Congo hemorrhagic fever virus
}

\author{
Connie Schmaljohn \\ Division U.S. Army Medical Research Institute of Infectious Diseases, \\ connie.schmaljohn@det.amedd.army.mil \\ Kristin Spik \\ United States Army Medical Research Institute of Infectious Diseases \\ Amy Shurtleff \\ United States Army Medical Research Institute of Infectious Diseases \\ Anita McElroy \\ United States Army Medical Research Institute of Infectious Diseases \\ Mary Guttieri \\ United States Army Medical Research Institute of Infectious Diseases, mary.guttieri@usda.gov
}

See next page for additional authors

Follow this and additional works at: https://digitalcommons.unl.edu/usarmyresearch

Schmaljohn, Connie; Spik, Kristin; Shurtleff, Amy; McElroy, Anita; Guttieri, Mary; and Hooper, Jay, "Immunogenicity of combination DNA vaccines for Rift Valley fever virus, tick-borne encephalitis virus, Hantaan virus, and Crimean Congo hemorrhagic fever virus" (2006). US Army Research. 291.

https://digitalcommons.unl.edu/usarmyresearch/291

This Article is brought to you for free and open access by the U.S. Department of Defense at DigitalCommons@University of Nebraska - Lincoln. It has been accepted for inclusion in US Army Research by an authorized administrator of DigitalCommons@University of Nebraska - Lincoln. 


\section{Authors}

Connie Schmaljohn, Kristin Spik, Amy Shurtleff, Anita McElroy, Mary Guttieri, and Jay Hooper 


\title{
Immunogenicity of combination DNA vaccines for Rift Valley fever virus, tick-borne encephalitis virus, Hantaan virus, and Crimean Congo hemorrhagic fever virus
}

\author{
Kristin Spik, Amy Shurtleff, Anita K. McElroy, Mary C. Guttieri, \\ Jay W. Hooper, Connie Schmaljohn* \\ Virology Division, United States Army Medical Research Institute of Infectious Diseases, \\ Fort Detrick, Frederick, MD 21702-5011, USA
}

Available online 22 August 2005

\begin{abstract}
DNA vaccines for Rift Valley fever virus (RVFV), Crimean Congo hemorrhagic fever virus (CCHFV), tick-borne encephalitis virus (TBEV), and Hantaan virus (HTNV), were tested in mice alone or in various combinations. The bunyavirus vaccines (RVFV, CCHFV, and HTNV) expressed Gn and Gc genes, and the flavivirus vaccine (TBEV) expressed the preM and E genes. All vaccines were delivered by gene gun. The TBEV DNA vaccine and the RVFV DNA vaccine elicited similar levels of antibodies and protected mice from challenge when delivered alone or in combination with other DNAs. Although in general, the HTNV and CCHFV DNA vaccines were not very immunogenic in mice, there were no major differences in performance when given alone or in combination with the other vaccines.
\end{abstract}

Published by Elsevier Ltd.

Keywords: DNA vaccine; Multiagent vaccine; Gene gun

\section{Introduction}

DNA vaccines offer tremendous promise for multiagent use. Among the advantages of DNA vaccines are their ease of construction, low risk, and absence of interference due to pre-existing immunity to a vector (e.g., vaccinia virus or adenovirus vectors). This technology, however, is still limited by effective means to deliver the DNA and a paucity of studies demonstrating efficacy in humans. Only a few studies have explored the potential of combination DNA vaccines in animals. We previously demonstrated that DNA vaccines for four highly pathogenic organisms, Venezuelan equine encephalitis virus, Ebola virus, Marburg virus, and Bacillus anthracis were able to elicit immune responses when given to animals individually or in combination [1]. In this report, we expand this area of research by testing combination DNA

\footnotetext{
* Corresponding author. Tel.: +1 301619 4103; fax: +1 3016192439

E-mail address: connie.schmaljohn@amedd.army.mil (C. Schmaljohn).
}

vaccines for four additional, highly pathogenic viruses, Hantaan virus (HTNV), tick-borne encephalitis virus (TBEV), Rift Valley fever virus (RVFV), and Crimean Congo hemorrhagic fever virus (CCHFV).

HTNV is the prototype of the Hantavirus genus of the family Bunyaviridae and is one of four hantaviruses known to cause hemorrhagic fever with renal syndrome (HFRS). HFRS caused by HTNV infection is found exclusively in Asia, with most cases occurring in China (reviewed in [2]). Hantaviruses are transmitted to humans by exposure to rodents' urine, feces, or saliva. The disease is characterized by fever and influenza-like symptoms, and in severe cases, shock and renal failure. A number of inactivated vaccines for HFRS have been developed and tested in Asia, but there is no vaccine currently approved by the U.S. Food and Drug Administration (reviewed in [3]).

CCHFV belongs to the Nairovirus genus of the family Bunyaviridae and causes a disease characterized by an abrupt onset of acute febrile illness that can progress to hemorrhage, renal failure, and shock. Mortality rates for $\mathrm{CCHF}$ have 
ranged from 10 to $70 \%$ in various outbreaks. Transmission usually occurs by tick-bite, but can also occur through contact with infected animal products or by person-to-person spread (reviewed in [4]). Evidence for the presence of CCHFV has been collected from South Africa through sub Saharan Africa, Eastern Europe, the Middle East and parts of China. No vaccine for CCHF has been developed.

RVFV, a member of the Phlebovirus genus of the family Bunyaviridae, is also is found in sub Saharan Africa, thus overlaps the endemic region of CCHF. RVFV is an important livestock pathogen causing abortion in pregnant animals, especially ewes, and a high mortality in newborn lambs, kids, and calves. RVF also impacts humans, as evidenced by a recent epidemic in Saudi Arabia and Yemen [5]. Disease in humans is characterized by an influenza-like febrile illness and can include complications, such as retinitis leading to blindness, and more rarely, encephalitis (reviewed in [6]). An inactivated preparation of RVFV has been developed and is currently available only under Investigation of New Drug (IND) status [7,8]. There are fewer than 3000 doses of the vaccine available, and preparation of new lots is problematic. Because RVFV is not endemic to the United States, and is such a virulent animal pathogen, its use is controlled. In addition, the facility that previously generated the virus for inactivation has closed. Consequently, alternative means for producing a vaccine have been explored. Toward this goal, a mutagenized, live-attenuated strain of RVFV, MP12, was developed and tested in mice, sheep, and cows [9-13]. This vaccine was quite promising in studies to assess its safety and efficacy; however, this vaccine has not yet been licensed.

TBEV is a member of the family Flaviviridae. Human disease is characterized first by a febrile illness, which is followed by CNS involvement in 20-30\% of cases (reviewed in [14]). Tick-borne encephalitis occurs in all countries of Central and Eastern Europe as well as in Scandinavia, France, Italy, Greece, and Albania (reviewed in [14]). A formalininactivated, chick embryo-derived vaccine was introduced into parts of Europe in 1976 and has resulted in a notable decline in disease. Despite the success of this vaccine, it suffers the disadvantages commonly associated with inactivated virus vaccines, such as the requirement for large-scale production and purification of a highly infectious human pathogen, the risk of incomplete inactivation of the virus, and the need to deliver the vaccine with adjuvant in a threeshot series [15]. Also, this vaccine is not licensed for use in the United States.

We previously reported constructing and testing DNA vaccines for HTNV and TBEV in animals. The HTNV vaccine expresses the complement of the medium (M) genome segment of the virus, which encodes the two viral glycoproteins $\mathrm{Gn}$ and $\mathrm{Gc}$ (referring to the amino- or carboxy-terminal encoded protein in the polyprotein precursor, and formerly designated G1 and G2). To gauge protective immunity, we use a hamster infection model in which we measure neutralizing antibody responses to the viral envelope glycoproteins after gene gun inoculation of the DNA vaccine, and then measure the absence of antibodies to the nucleocapsid protein (which is not a component of the vaccine) after challenge with the hantavirus [16]. We have shown that the HTNV DNA vaccine induces high levels of neutralizing antibodies in hamsters and completely protects them from challenge (i.e., confers sterile immunity) [16,17]. In addition, we found that in monkeys, the vaccine induces high levels of neutralizing antibodies, which persist for many months after vaccination $[17,18]$.

The TBEV DNA vaccine expresses the preM and $\mathrm{E}$ genes of a central European isolate of TBEV (Hypr strain) [19]. We previously demonstrated that this vaccine confers protective immunity to mice for at least 1 year after vaccination [19], and elicits high levels of neutralizing antibodies in monkeys [20]. Passive transfer of sera from vaccinated monkeys protects mice from lethal challenge with TBEV [20].

DNA vaccines for RVFV have not yet been reported. We demonstrated in earlier studies that injecting mice with baculovirus-expressed RVFV M segment products elicited protective immunity [21]. Similarly, infecting mice with a recombinant vaccinia virus expressing the $\mathrm{M}$ segment of RVFV elicited protection from RVFV challenge [22]. In addition, passive transfer of neutralizing antibodies to either Gn or Gc protected mice from challenge with RVFV [23]. Consequently, the DNA vaccines that we developed for the studies reported here, express the $\mathrm{M}$ genome segment of the virus.

To date, there have been no reports of recombinant DNA vaccines for CCHFV. As for other viruses in the family, we surmised that the M segment expression products would be the most likely candidates for eliciting protective immunity. Unlike those of other viruses in the family, however, the $\mathrm{M}$ segment precursor of CCHFV Gn and Gc has been found to undergo at least two post-translational proteolytic cleavage events. A precursor of the Gn glycoprotein is cleaved at the conserved motifs RSKR and RRLL, potentially releasing a highly variable mucin-like domain at its amino terminus, and a second $\mathrm{N}$-terminal domain of approximately $35 \mathrm{kDa}$ (P35) as well as the $37 \mathrm{kDa}$ mature Gn [24,25]. An $85 \mathrm{kDa}$ precursor of Gc has also been described [24]; however, the cleavage events leading to the mature $75 \mathrm{kDa}$ Gc protein have not been determined. The importance of these precursor polypeptides in viral morphogenesis or pathogenesis is not known. For the studies reported here, we generated a DNA vaccine construct expressing the entire $M$ genome segment of CCHFV.

In a recent study, monoclonal antibodies (MAbs) to CCHFV Gc, but not to $\mathrm{Gn}$, neutralized virus in plaque reduction neutralization tests (PRNT) [26]. However, MAbs directed against $\mathrm{Gn}$ were generally more effective at protecting mice from a lethal CCHFV challenge than MAbs to Gc when administered either $24 \mathrm{~h}$ before or after infection even though these Gn MAbs did not neutralize in the cell-culture assays. In addition, not all of the Gc MAbs that neutralized CCHFV infection in vitro conferred protection in vivo. Thus, there was not a strict correlation between in vitro neutralization and in vivo protection [26]. 
Here, we report constructing and testing in mice two RVFV M segment DNA vaccines and an M segment DNA vaccine for CCHFV alone or combined with the DNA vaccines for HTNV and TBEV.

\section{Materials and methods}

\subsection{DNA vaccine construction}

All genes were inserted into pWRG7077, a plasmid containing a cytomegalovirus immediate early promoter and a kanamycin resistance gene [19] or a slightly modified version of that plasmid [17].

The construction of the TBEV vaccine and the HTNV vaccine were reported earlier $[17,19]$.

We constructed two DNA vaccines for RVFV, both of which express the viral $\mathrm{M}$ genome region encoding the envelope glycoproteins, Gn and Gc, but which differ in the amount of nonstructural $\mathrm{M}$ segment (NSm) coding information that is included. To generate $\mathrm{RVFV}_{+\mathrm{NSm}}$ and $\mathrm{RVFV}_{-\mathrm{NSm}}$, PCR primers were designed to incorporate Not 1 and $E c o$ R 1 sites for cloning into pWRG7077 containing a multiple cloning site inserted between the Not 1 and $B g l I I$ sites of the plasmid. Segments were amplified from plasmids used previously for constructing recombinant baculoviruses, pAcYM1R2 and pAc373-R4 [21], with the following primers: Not1(Forward) +NSM5'GTCAGTCAGTCAGTCAGCGGCCGCATGATTGAAGGAGCT3'; Not1(Forward)-NSM5'GTCAGTCAGTCAGTCAGCGGCCGCATGGCAGGGATTGCA3'; EcoR1(Reverse) 5'CAGTCAGTCAGTCAGTGAATTCACCACCCCAAATTAC $3^{\prime}$.

The DNA vaccine for CCHFV was constructed by excising the Not 1 cassette containing CCHFV, strain 10200, M segment sequence from a pBluescript construct kindly provided by Dr. Michael Parker. The resulting fragment was then cloned into the modified pWRG7077 using the Not 1 site. The sequence of the clone was confirmed and expression was checked as described below.

\subsection{Transient expression assays}

Methods used for measuring transient expression of the DNA vaccines were described earlier [19]. Briefly, for each assay, $5 \mu \mathrm{g}$ of each DNA vaccine was transfected into monolayers of COS cells plated in 6-well plates (Costar) by using FuGENE6 (Roche) reagent (Gibco) according to the manufacturer's directions. At $24 \mathrm{~h}$ after transfection, the medium was removed from the wells, the cells were incubated with cysteine and methionine-deficient medium, then radiolabeled with 35S Promix (methionine and cysteine, Amersham). Cells were lysed on ice using Zwittergent 3-14 (Calbiochem-Behring) lysis buffer and cellular nuclei removed by centrifugation. Radiolabeled lysates were precipitated with specific antibodies to each virus and visualized by phosphoimaging after electrophoresis in $4-12 \%$
Bis-Tris pre-cast gels (Invitrogen) run in MOPS buffer (Invitrogen).

\subsection{Preparation of gene gun cartridges, vaccination, and challenge of mice with RVFV, or TBEV}

Plasmid DNA was precipitated onto the outside surfaces of gold beads (approximately $2 \mu \mathrm{M}$ in diameter) as described previously [27]. The DNA loads were $0.5-1 \mu \mathrm{g} / \mathrm{mg}$ of gold. For studies involving the genetic adjuvants, the $\mathrm{RVFV}_{+\mathrm{NSm}}$ DNA vaccine was mixed with plasmids individually encoding the alpha and beta subunits of cholera toxin (CT) or the alpha and beta subunits of a labile enterotoxin from $E$. coli (LT) [28] in a ratio of 2:1:1 and the mixture was precipitated onto gold beads. The DNA-coated gold was dried on the inside walls of Tefzel tubing, which was then cut into 0.5 in sections. Female BALB/c mice (approximately 4- to 6-weeks old) were vaccinated by using the XR-1 gene gun (Powderject Vaccines, Inc.) as reported previously [29]. For challenge studies, mice were transferred to a biosafety level 3 (RVFV) or level 4 (TBEV) containment area and challenged by intraperitoneal inoculation of approximately $100 \mathrm{LD}_{50}$ of TBEV (strain Hypr) or RVFV (strain ZH501). Mice were observed for signs of illness and weighed daily for at least 21 days.

\subsection{Plaque reduction neutralization tests}

Sera from vaccinated mice were incubated at $56^{\circ} \mathrm{C}$ for 30 min, then diluted 1:40-1:1280 in EMEM with 10\% fetal bovine serum (FBS). A viral stock of known titer was then diluted to $1 \times 10^{3}$ plaque forming units $(\mathrm{pfu}) / \mathrm{ml}$ in either EMEM with $10 \%$ FBS or Hanks Balanced Salt Solution (HBSS) $+5 \%$ FBS. An equal volume of diluted virus was then added to each serum dilution and also to an EMEM-only control. The tubes were incubated at $4{ }^{\circ} \mathrm{C}$ overnight. The following day, $200 \mu \mathrm{l}$ of the virus/serum mixture was added to duplicate wells containing 3- to 7-day-old Vero or Vero E6 monolayers in 6-well plates. The plates were incubated for 90 min at $37^{\circ} \mathrm{C} / 5 \% \mathrm{CO}_{2}$ with gentle rocking and shaking every $15 \mathrm{~min}$ to distribute the inoculum over the monolayer. At the end of the incubation period, an agarose overlay was prepared as follows: for every $100 \mathrm{ml}$ of overlay needed, $0.6 \mathrm{~g}$ of SeaKem ME agarose was added to $34 \mathrm{ml}$ of water, autoclaved, then held in a $60{ }^{\circ} \mathrm{C}$ water bath. The following were then mixed together, warmed, and then added to the agarose: $50 \mathrm{ml} 2 \times$ EMEM, $10 \mathrm{ml}$ of FBS, $4 \mathrm{ml} \mathrm{L}$-glutamine $(200 \mathrm{mM}), 1 \mathrm{ml} 100 \times$ nonessential amino acids, $1 \mathrm{ml}$ of penicillin/streptomycin, and $1 \mathrm{ml}$ of Amphotericin B. Each well was overlaid with $2 \mathrm{ml}$ of the overlay mixture. The plates were then incubated for 3-7 days at $37^{\circ} \mathrm{C} / 5 \% \mathrm{CO}_{2}$, after which $2 \mathrm{ml}$ of secondary overlay was added to each well. This overlay was identical to the primary overlay with the exception that only $5 \mathrm{ml}$ of FBS and $5 \mathrm{ml}$ of neutral red solution (Gibco) were added. Plaques that appeared during the next 2-3 days were counted and the neutralizing antibody titers were 
calculated as a reciprocal of the highest dilution resulting in a $50 \%$ or $80 \%$ reduction of the plaque number as compared to the virus-only control wells.

\section{Results}

\subsection{Construction of RVFV DNA vaccines and demonstration of expression in cell culture}

Two DNA vaccines for RVFV were constructed, which differed only in the amount of nonstructural $M$ segment coding information that was included. As described earlier, the $\mathrm{M}$ genome segment + sense RNA has four potential translation initiation codons (ATG) upstream of the coding information for the amino-terminal glycoprotein, $\mathrm{Gn}$, and the carboxy-terminal glycoprotein, Gc [30]. Initiation at the second ATG produces a $14 \mathrm{kD}$ NSm protein as well as $\mathrm{Gn}$ and Gc. Initiation at the 4th ATG results in only Gn and Gc. We previously demonstrated that baculovirus-expressed proteins derived from genes in which translation initiates at the 2nd ATG (in this report referred to as $\mathrm{RVFV}_{+\mathrm{NSm}}$ ) or from the 4 th ATG (in this report referred to as $\mathrm{RVFV}_{-\mathrm{NSm}}$ ) were immunogenic in mice, [21]. In this study, we evaluated both constructs to see if the presence of the additional NSm product would contribute to or detract from the immunogenicity of the DNA vaccine.

To assay for gene expression, we transfected cultured cells with the two RVFV DNA vaccine constructs, then radiolabeled and immune precipitated expression products with polyclonal sera to RVFV. Both constructs produced RVFV Gn and Gc (Fig. 1A). A small amount of the NSm protein (formerly $14 \mathrm{kDa}$ protein) also was precipitated from the $\mathrm{RVFV}_{+\mathrm{NSm}}$ construct (Fig. 1A). Both constructs expressed $\mathrm{Gn}$ and Gc, although it appeared that there was a slightly higher level of expression with the $\mathrm{RVFV}_{-\mathrm{NSm}}$ construct (Fig. 1A).

\subsection{DNA vaccine for $C C H F V$}

The DNA vaccine for CCHFV was constructed to express the entire $M$ genome segment. Transfecting cells, radiolabeling, and immune precipitating the expression products revealed products of the expected sizes for mature Gn $(37 \mathrm{kDa})$ and $\mathrm{Gc}(75 \mathrm{kDa})$ and a larger product, which might be the $140 \mathrm{kDa}$ precursor of Gn [24] (Fig. 1B).

\subsection{Immunogenicity of individual and combination DNA vaccines for $R V F V_{+N S m}, C C H F V, H T N V$, and TBEV in mice}

Groups of mice were vaccinated by gene gun three times at approximately 4-week intervals with each DNA vaccine or with a combination of DNA vaccines for RVFV, CCHFV, HTNV, and TBEV. At each dosing, mice in the individual vaccine groups received four gene gun administrations of the DNA coated on gold (approximately $10 \mu \mathrm{g}$ of DNA). Mice in the combination groups received one gene gun administration of each of the four DNA vaccines (approximately $2.5 \mu \mathrm{g}$ of each DNA, for a total of $10 \mu \mathrm{g}$ of DNA). Mice are lethal challenge models for both RVFV and TBEV, consequently, challenges with TBEV or RVFV, but not with HTNV or $\mathrm{CCHFV}$ were performed.

As expected, the mice receiving only the TBEV DNA vaccine developed strong neutralizing antibody responses (Fig. 2A). After challenge with TBEV, all (8/8) of these mice remained healthy throughout 22 days of observation, as evidenced by the absence of apparent illness, and no weight loss (Fig. 2C). Mice that received all four of the DNA vaccines also developed strong neutralizing antibody responses to TBEV
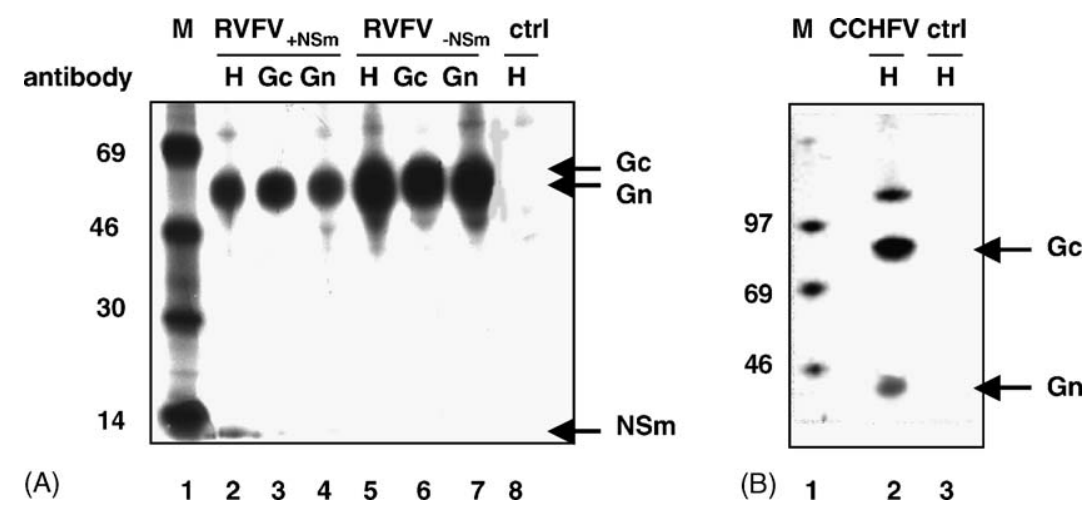

Fig. 1. Immune precipitation of radiolabeled expression products of the RVFV and CCHFV DNA vaccines. The DNA vaccines were transfected into cultured cells, the expression products radiolabeled, and immune precipitated using antibodies to RVFV or CCHFV. (A) Two RVFV M genome segment DNA vaccines were compared. The RVFV_NSm construct contains coding information only for the two envelope glycoproteins, Gn and Gc. In addition to Gn and Gc, the $\mathrm{RVFV}_{+\mathrm{NSm}}$ construct also encodes a $14 \mathrm{kDa}$ nonstructural protein (NSm). A plasmid with no insert was used as a negative control (ctrl, lane 8 ). Antibodies used for immune precipitation were hyperimmune mouse ascitic fluid to RVFV (H, lanes 2, 5 and 8); a monoclonal antibody to Gc (Gc, lanes 3 and 6); or a monoclonal antibody to Gn (Gn, lanes 4 and 7). (B) Expression of a DNA vaccine containing the coding region of the M genome segment of CCHFV was evaluated by immune precipitation using a hyperimmune mouse serum to CCHFV (H, lane 2). Products from a plasmid with no insert (ctrl, lane 3 ) were precipitated by the same serum. Size markers were included to determine approximate molecular weights of expression products (M, lane 1 both figures). 


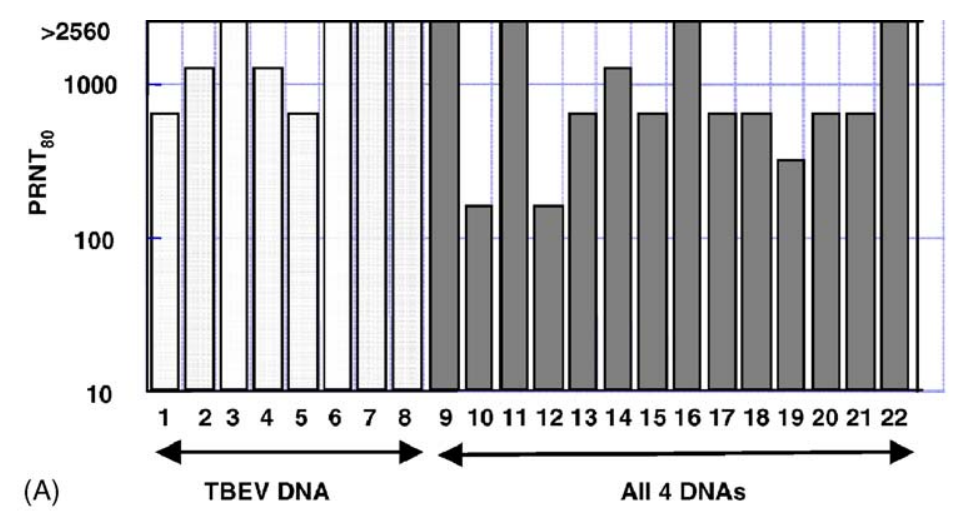

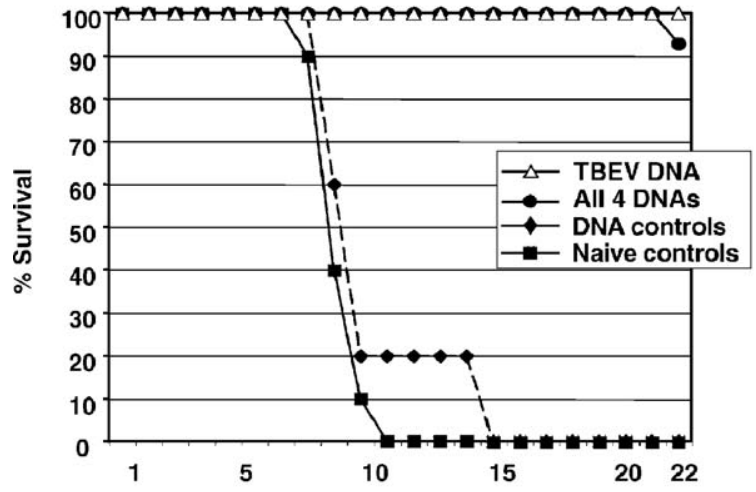

(B) Days After Challenge

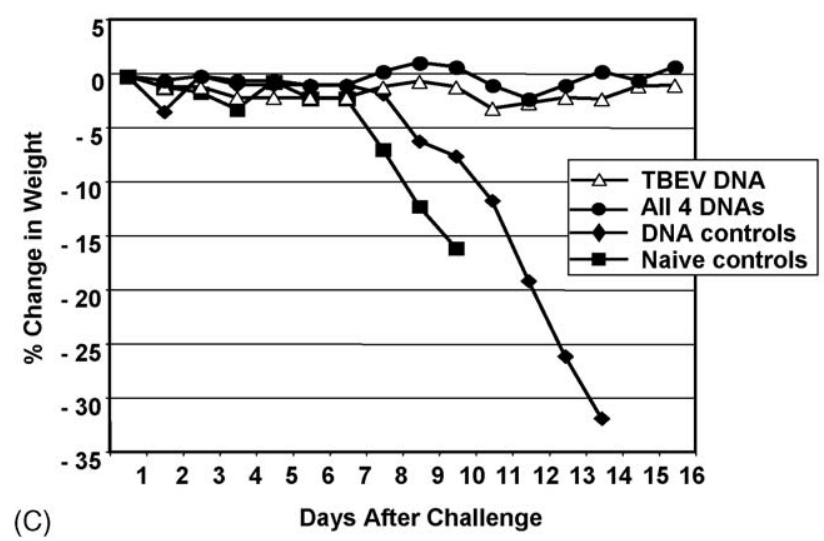

Fig. 2. Neutralizing antibody responses and protection from challenge of mice vaccinated with the TBEV DNA vaccine alone (TBEV DNA) or in combination with the HTNV, RVFV + NSm and CCHFV DNA vaccines (All four DNAs). (A) PRNT80 titers of mice after three inoculations with the TBEV DNA vaccine (lanes 1-8) or all of the DNA vaccines (lanes 9-22). (B) Survival of vaccinated mice and controls receiving empty plasmid (DNA controls) or no vaccine (naïve controls). (C) Percent change in weight per group of mice after challenge with TBEV.

(Fig. 2A), and upon challenge, 13/14 mice survived. We suspect that the one death was not due to the TBEV infection, as there was no sign of illness before death and there was no weight loss in the group of mice that included this mouse (Fig. 2C and data not shown).

Although we have not developed an adult mouse model for HTNV or for CCHFV infections, we were able to measure antibody responses to the vaccine in the vaccinated mice. After three gene gun vaccinations with the HTNV DNA vaccine, we found that 3 of 10 mice in the single vaccine group and 9 of 26 mice in the multiagent groups developed neutralizing antibodies to HTNV (PRNT 50 1:40-1:80 in the single group, 1:40-1:160 in the multiagent group, data not shown). Pools of sera from mice with or without neutralizing antibodies were both able to immune precipitate radiolabeled, expressed HTNV Gn and Gc, although Gc was more apparent (Fig. 3A).

Only about half of the mice that received the CCHFV DNA vaccine, either alone or combined with other vaccines, developed neutralizing antibodies to CCHFV $\left(\mathrm{PRNT}_{50}<1: 40-1: 160\right)$. Immune precipitation of radiolabeled proteins using pooled sera from mice given the individual or combined DNAs revealed antibody responses to Gn and Gc (Fig. 3B).
Unexpectedly, mice vaccinated with the $\mathrm{RVFV}_{+\mathrm{NSm}}$ vaccine did not develop neutralizing antibody responses, and only three of nine mice in the individual group and 3 of 14 mice in the multiagent group survived challenge with RVFV. To determine if a non-neutralizing antibody response was induced in vaccinated mice, we transfected cells with the $\mathrm{RVFV}_{+\mathrm{NSm}}$ DNA vaccine, then radiolabeled, and immune precipitated expression products using sera collected after the third vaccination. We could not detect signals for $\mathrm{Gn}$ and Gc in any of the vaccinated mice before challenge, although a control immune serum did precipitate both proteins (data not shown).

Because of the poor performance of the $\mathrm{RVFV}_{+\mathrm{NSm}} \mathrm{DNA}$ vaccine, we carried out two additional experiments. In one of the experiments, we re-evaluated the $\mathrm{RVFV}_{+\mathrm{NSm}}$ DNA vaccine in conjunction with two gene gun-delivered adjuvants, and in the second experiment, we evaluated our other RVFV construct, $\mathrm{RVFV}_{-\mathrm{NSm}}$.

For the adjuvant experiment, we co-administered the $\mathrm{RVFV}_{+\mathrm{NSm}}$ vaccine with plasmids expressing the alpha and beta subunits of either $E$. coli labile enterotoxin (LT) or cholera toxin (CT) to groups of mice. Co-administration of these genetic adjuvants has been shown to augment the Th1 cytokine responses (gamma interferon) in mice to multiple 


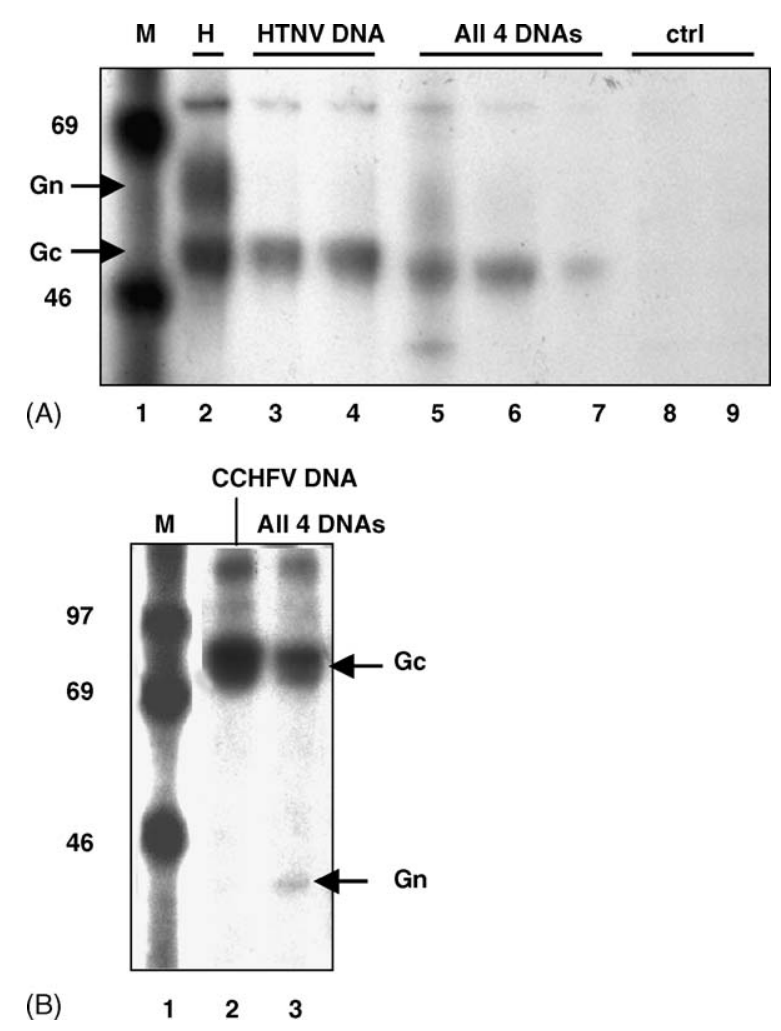

Fig. 3. Immune precipitation of radiolabeled, expressed HTNV or CCHFV proteins using sera from vaccinated mice. (A) Precipitation of HTNV expression products by hyperimmune mouse ascitic fluid to HTNV (H, lane 2$)$ or with pooled sera from mice given only the HTNV DNA vaccine (HTNV DNA, lanes 3,4) or in combination with DNA vaccines for CCHFV, $\mathrm{RVFV}_{+\mathrm{NSm}}$, and TBEV (All four DNAs, lanes 5, 6, 7). Pooled sera from control mice vaccinated with a plasmid with no insert served as negative controls (ctrl, lanes 8, 9). (B) Pooled sera from mice vaccinated with the CCHFV vaccine only (CCHFV DNA, lane 2) or with the vaccine given in combination with DNA vaccines for HTNV, TBEV and $\mathrm{RVFV}_{+\mathrm{NSm}}$ (All four DNAs, lane 3). Protein size markers (M) are in lane 1 of both figures.

viral antigens when co-delivered with DNA vaccines. In addition, both adjuvants also increased antibody responses and Th2 cytokine responses (interleukin 4) to certain antigens tested [28].

In our experiment, groups of mice were vaccinated four times by gene gun with the $\mathrm{RVFV}_{+\mathrm{NSm}}$ DNA by itself or mixed with the genetic adjuvants. Control groups were vaccinated with either the empty plasmid or this plasmid mixed with the genetic adjuvants. Mice were bled 3 weeks after the last vaccination and sera were evaluated by PRNT and immune precipitation. The mice were then challenged with $100 \mathrm{LD}_{50}$ of RVFV. PRNT 50 titers of $1: 40$ or $1: 80$ were measured in four of nine mice given the $\mathrm{RVFV}_{+\mathrm{NSm}}$, but no neutralizing antibodies were detected in mice receiving the vaccine combined with either of the adjuvants. Survival to challenge in the $\mathrm{RVFV}_{+\mathrm{NSm}}$ group, CT group, or LT group, was $2 / 9,3 / 10$ and 5/10 mice, respectively (Fig. 4A). Thus, the $\mathrm{RVFV}_{+\mathrm{NSm}}$ DNA vaccine was poorly immunogenic with or without the adjuvants.
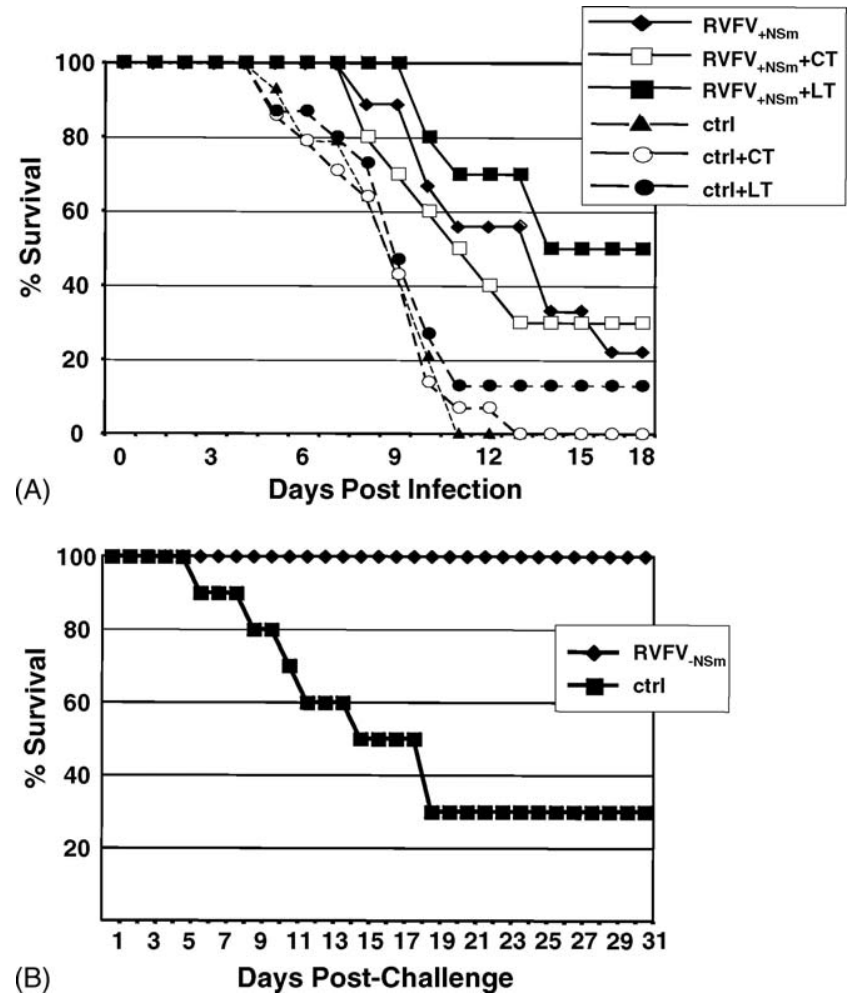

Fig. 4. Survival of mice vaccinated with the RVFV DNA vaccines. (A) Groups of mice were inoculated four times with the $\mathrm{RVFV}_{+\mathrm{NSm}}$ vaccine alone, or mixed with genetic adjuvants encoding the alpha and beta subunits of cholera toxin (CT) or E. coli labile enterotoxin (LT). Control mice received a plasmid with no insert (ctrl) or the empty plasmid mixed with the CT or LT genetic adjuvants. Mice were challenged with 100 LD $_{50}$ of RVFV and survival monitored for 30 days. No changes in survival after the 18 days shown were noted. (B) Mice were vaccinated three times with the $\mathrm{RVFV}_{-\mathrm{NSm}}$ DNA vaccine or a control plasmid with no insert (ctrl), then challenged with 100 LD $_{50}$ of RVFV. Survival was monitored for 31 days after challenge.

In contrast to the $\mathrm{RVFV}_{+\mathrm{NSm}}$ vaccine, the $\mathrm{RVFV}_{-\mathrm{NSm}}$ vaccine elicited neutralizing antibodies to RVFV (PRNT 50 1:40-1:320) in all 10 mice receiving three gene gun vaccinations and all mice survived challenge with RVFV (Fig. 4B). Although 3 of 10 mice in the control group survived challenge, all three showed signs of extreme illness, whereas the $\mathrm{RVFV}_{-\mathrm{NSm}}$-vaccinated mice appeared healthy throughout the observation period.

\subsection{Immunogenicity of individual and combination $D N A$ vaccines for $R V F V_{-N S m}$, HTNV, and TBEV in mice}

To evaluate the RVFV -NSm vaccine when given in combination with two of the other vaccines, we vaccinated groups of mice three times at 3-week intervals with two gene gun administrations/mouse of the individual DNAs (total $5 \mu \mathrm{g}$ of DNA) or with two gene gun inoculations per mouse of each of the three DNA vaccines ( $5 \mu \mathrm{g}$ of each vaccine).

The $\mathrm{RVFV}_{-\mathrm{NSm}}$ vaccine elicited strong neutralizing antibody responses in all but one mouse in the individual group 

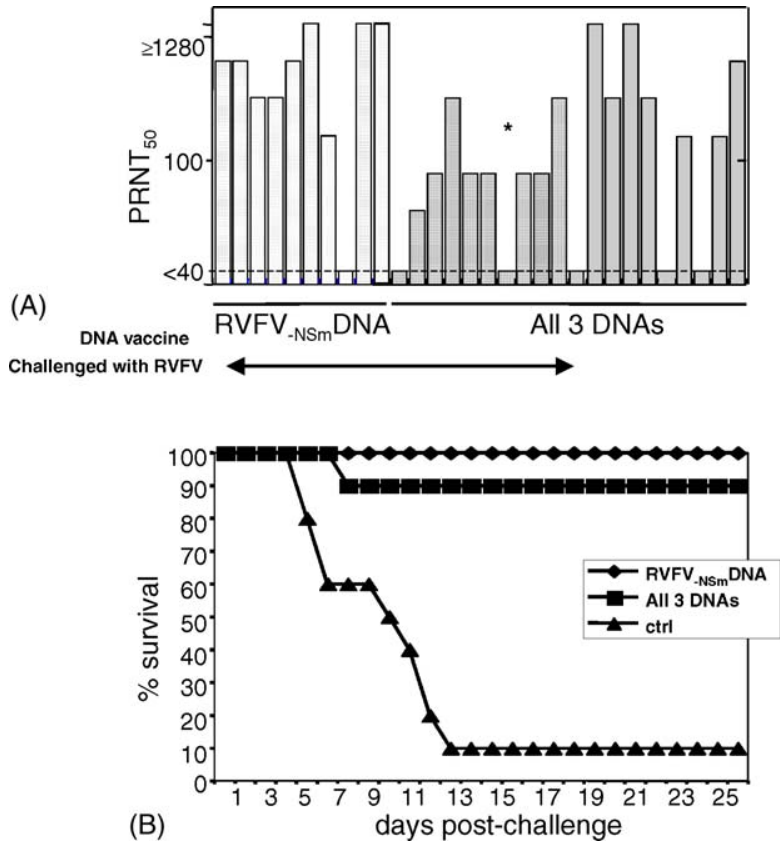

Fig. 5. Neutralizing antibody responses and survival to challenge of mice vaccinated with the $\mathrm{RVFV}_{-\mathrm{NSm}}$ DNA vaccine. (A) $\mathrm{PRNT}_{50}$ titers were determined for mice inoculated three times with the $\mathrm{RVFV}_{-\mathrm{NSm}}$ DNA vaccine alone (RVFV -NSm DNA, white bars) or in combination with DNA vaccines for TBEV and HTNV (All three DNAs, grey-shaded bars). Data from mice challenged with RVFV are indicated by the stippling in the white or grey bars. An asterisk indicates the mouse that died after challenge with RVFV. (B) Mice vaccinated with only the RVFV - NSm DNA vaccine, with all three DNA vaccines, or with a control plasmid with no insert (ctrl) were challenged with $1000 \mathrm{LD}_{50}$ of RVFV and survival was monitored for 30 days, with no changes observed after the 26 days shown.

(PRNT 50 1:160-1:1280) (Fig. 5A). All 10 mice survived challenge with RVFV. Of the mice receiving the RVFV vaccine combined with the TBEV and HTNV vaccines, 15 of 20 had neutralizing antibody responses of 1:40 or greater (Fig. 5A). Of the 10 mice challenged with RVFV in the multiagent group, 9 survived, with the single death occurring in a mouse with a $<1: 40$ neutralizing antibody response (Fig. 5A and B). All control mice became ill and 9 of 10 died (Fig. 5B).

As in the earlier experiment, mice vaccinated with the TBEV DNA vaccine alone or in combination with other vaccines developed strong neutralizing antibody responses to TBEV (Fig. 6) and all mice survived challenge.

Similar to results obtained with HTNV in the four-vaccine study, 5 of 10 mice in the individual group, and 4 of 17 mice in the multiagent groups developed neutralizing antibody responses to HTNV (PRNT 50 1:40-1:160). In the earlier study, we only assayed pooled sera by immune precipitation to determine if the vaccinated mice had antibodies to HTNV. In this study, we assayed each mouse's serum in the individual group, and pools of sera from five mice in the multiagent groups. We found that all samples assayed precipitated radiolabeled HTNV envelope glycoproteins, and that there was no apparent difference between the signals observed when

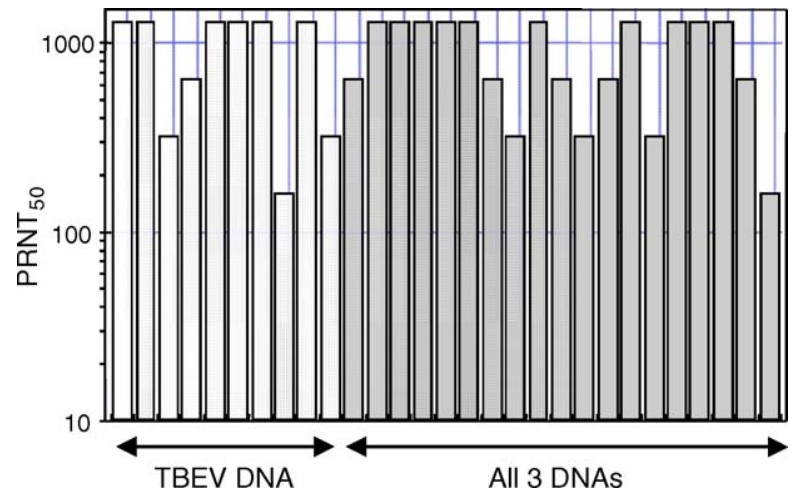

Fig. 6. Neutralizing antibody responses of vaccinated mice to TBEV. PRNT50 titers of sera from mice vaccinated with the TBEV DNA vaccine alone (TBEV DNA, white bars) or combined with the $\mathrm{RVFV}_{-\mathrm{NSm}}$ and the HTNV DNA vaccines (All three DNAs, grey-shaded bars) were determined. The stippling of the white bars and grey bars indicates data from mice challenged with TBEV.

proteins were precipitated with sera from mice with or without neutralizing antibodies (data not shown).

\section{Discussion}

We constructed DNA vaccines for RVFV and CCHFV and tested their immunogenicity in mice. We further compared these DNA vaccines in mice in combination with each other and with DNA vaccines that we previously engineered for HTNV and TBEV.

RVFV has a complicated natural expression strategy, with at least two in-frame translation initiation codons used in normal infections. Initiation at the first in-frame ATG results in $78 \mathrm{kDa}$ protein representing a fusion of the entire preglycoprotein coding region and $\mathrm{Gn}$, as well as Gc. Initiation from this ATG allows the use of a glycosylation site within the preglycoprotein coding region, but this glycosylation is apparently unimportant for cleavage of the $78 \mathrm{kDa}$ protein to yield NSm and Gn [31,32]. Translation from the second ATG, which is found 37 amino acids downsteam from the first, yields a $14 \mathrm{kDa} \mathrm{NSm}$ protein and $\mathrm{Gn}$ and Gc [31,32]. The glycosylation site, which is used during translation from the first ATG, is not used in translation from the second ATG [32]. Although it is not clear if the fourth in-frame ATG is used by RVFV during infection, it can be used to generate $\mathrm{Gn}$ and Gc in a variety of expressions systems ([21,30-33], this report).

In our studies, we found that the $\mathrm{RVFV}_{-\mathrm{NSm}}$ construct, which uses the fourth in-frame translation initiation codon, was highly immunogenic in mice and elicited protective immunity. In contrast, the $\mathrm{RVFV}_{+\mathrm{NSm}}$ vaccine, which uses the second in-frame codon, was not immunogenic. This finding was unexpected in that both the constructs expressed in cell culture and produced apparently similar amounts of $\mathrm{Gn}$ and Gc. We do not think that this finding reflects a technical difficulty with the $\mathrm{RVFV}_{+\mathrm{NSm}}$, in that similar results were 
obtained in two separate experiments, one with the CT and LT genetic adjuvants, and one without them. The adjuvants did not improve the antibody responses elicited to the $\mathrm{RVFV}_{+\mathrm{NSm}}$ vaccine, which was not entirely unexpected. In earlier studies, influences of both sets of vectors on antibody responses were antigen dependent and ranged from no effect to sharp reductions in the immunoglobulin G1 (IgG1)-to-IgG2a ratios [28]. Here, we did not assess the antibody isotypes in the sera of vaccinated mice, thus we do not know if the adjuvants shifted the responses from the normal Th2-type response that we observe after gene gun vaccination of mice toward a more Th1-type response. The LT adjuvant may have provided some benefit in that there was a small improvement in survival among mice that received the LT subunits along with the $\mathrm{RVFV}_{+\mathrm{NSm}}$ vaccine (5 of 10 survived) as compared to those that only received the $\mathrm{RVFV}_{+\mathrm{NSm}}$ vaccine (two of nine survived). We did not pursue this finding further, because of the poor performance of the vaccine itself.

Earlier studies demonstrated that vaccinia virus-expressed RVFV genes, which either included or eliminated the preglycoprotein coding region, trafficked normally through the Golgi [33]. A more recent study, with a T7 expression system, confirmed these earlier results and further demonstrated that the Golgi localization signal is found in $\mathrm{Gn}$ in a region consisting of a 20 amino acid transmembrane domain and the adjacent 28 amino acids of the cytosolic tail [34]. Although we do not have an explanation as to why the $\mathrm{RVFV}_{+\mathrm{NSm}}$ construct would behave differently in mice than in cell culture, the unexpected absence of immunogenicity for a construct is something we have observed before in our studies of hantavirus DNA vaccines. We showed that although DNA vaccines for two hantaviruses, HTNV and Seoul virus, are highly immunogenic in hamsters, a DNA vaccine for another hantavirus, Andes virus (ANDV), which was constructed the same way as the other two vaccines, failed to elicit immune response in hamsters [18]. Interestingly, however, this ANDV DNA vaccine was able to elicit high levels of neutralizing antibodies in nonhuman primates [18]. Thus, there clearly can be differences not only between cell-culture expression and induction of immunity in animal models, but also among different animal models.

Like RVFV, CCHFV uses a complicated and unique, expression strategy. CCHFV and other nairoviruses, differ from other animal viruses in the family in that they use posttranslational processing as well as co-translational processing to generate their mature envelope glycoproteins. There is currently no information available to relate the processing events to viral pathogenesis; therefore, in our studies, we expressed the entire $M$ segment coding region. In cell-culture expression assays, correct processing appears to have occurred, in that we were able to detect polypeptides of the expected sizes for both Gn and Gc. In addition, we were able to demonstrate that the CCHFV DNA vaccine elicited neutralizing antibodies in some of the vaccinated mice as well as antibodies able to immune precipitate radiolabeled expression products. Unfortunately, there is no known challenge model for CCHFV, thus we were unable to determine if our vaccine offered protective immunity. We currently are attempting to generate a CCHFV that is able to kill adult mice, and if we are successful, will retest this vaccine for protective efficacy. In addition, we are preparing CCHFV constructs that express each of the mature glycoproteins separately and will compare their immunogenicity to that of the DNA vaccine described in this report.

Adult mice are not particularly useful models for HTNV infection. Newborn mice have been found to suffer fatal neurological disease after intracerebral [35,36] or intraperitoneal injection of HTNV [37], but immunocompetent adult mice generally clear the virus. One recent report, however, found that adult mice can also suffer neurological disease and death after intraperitoneal injection of HTNV [38]. It is not clear why these results differ from those of earlier studies, but there is a possibility that the virus used in the challenges had undergone some minor mutations, as evidenced by nucleotide and amino acid sequence changes that the authors noted. As we did not have access to this particular HTNV stock during our studies, we did not attempt to use it for challenge; however, it would be interesting to compare that viral stock to our own in mice.

In our studies, we found that mice did not mount strong neutralizing antibody responses to our HTNV DNA vaccine. In contrast, this same HTNV DNA vaccine was able to elicit strong neutralizing antibody responses in hamsters and monkeys [16-18,39]. Vaccinated mice did appear to develop antibodies to HTNV detectable by immune precipitation, and there were no obvious differences in the results from the individual and multiagent groups. For a more comprehensive evaluation of this HTNV DNA vaccine in a multiagent format, it will likely be necessary to perform the HTNV portions of the study either in hamsters or in nonhuman primates.

Although mice were not a good models for the HTNV or CCHFV DNA vaccines, they were excellent models for the $\mathrm{RVFV}_{-\mathrm{NSm}}$, and TBEV DNA vaccine studies. Here and in earlier studies, we found that both mice and nonhuman primates develop high levels of neutralizing antibodies to TBEV after DNA vaccination [19,20]. Consequently, a comparison of the immune responses of mice to the RVFV and TBEV vaccines in combination experiments can probably provide the most insight into the potential for multiagent DNA vaccines for these four viruses in mice.

In both the four DNA and the three DNA vaccine experiments, the TBEV DNA vaccine elicited strong neutralizing antibody responses and protective immunity when given alone or combined with other DNA vaccines. There did appear to be a trend toward slightly higher responses in the individual group versus the multiagent groups in the first experiment; however, in the second experiment the geometric mean titers of the neutralizing antibody responses for the groups were nearly the same. For the $\mathrm{RVFV}_{-\mathrm{NSm}}$ vaccine, in the second study, however, there still appeared to be a trend toward a better response in the single than in the multiagent group with overall consistency of developing neutralizing 
antibodies as well as higher neutralizing antibody titers seen in mice in the individual group as compared to the multiagent groups.

Despite these apparent trends, in general, we found no evidence for drastic diminution of immunogenicity when the vaccines were given together as opposed to individually. Although to our knowledge, there are no reports of interference among unrelated gene products, such as those in our study, there have been reports of interference when two or more genes from the same organism are used together as DNA vaccines. For example, when DNA vaccines expressing the L1R and A33R of vaccinia virus are delivered together into the same cells of a mouse by gene gun (i.e., are coated on the same gold beads), the L1R response is greatly reduced as compared to when L1R is given by itself. However, if the DNAs are delivered to different cells of the mouse (DNAs coated on different gold beads), strong responses are elicited to both gene products [40]. The authors hypothesized that the interference might be due to A33R-specific antibodies elicited by the initial vaccination, causing a lysis of A33R-expressing cells during subsequent boosts and, in doing so, diminishing the boosting effect [40]. In another study, five plasmids expressing different malaria genes were injected into mice and immune responses measured in mice given each plasmid separately or as a mixture of all five. Although the mixture induced higher levels of antibodies against whole parasites than did the individual plasmids, there were decreased amounts of antibodies to the individual gene products. In addition, T-cell responses were generally lower when the mixture was given [41]. Other studies, however, have not shown interference when similar genes are delivered (e.g., multiple HIV-1 genes from different clades [42]); or when two genes from the same virus are given (e.g., hepatitis B surface and core genes [43]).

One obvious practical problem with drawing inferences concerning a trend toward slightly lower immunogenicity of our DNA vaccines when given in combination as opposed to separately revolves around the limitations of gene gun delivery of DNA to small animals, such as mice. With current technology, only about 2-3 $\mu$ g of DNA can be delivered by a single gene gun administration. In a multiple vaccine study, such as ours, it is only possible to give one or two non-overlapping administrations of each vaccine per dose, because of the small surface area available on mice. In our first experiment, therefore, we were only able to give one administration of each vaccine to the mice receiving all four vaccines, while the individual group mice received four administrations per dose. In the second experiment, we attempted to normalize this by reducing the number of DNA vaccines given to three instead of four, so that we could give two administrations of each vaccine to mice in the multiagent group as well as in the individual groups. Even so, two administrations may not be sufficient to provide optimal immunogenicity with these vaccines. It is possible that more administrations per dose (as we are able to do in larger animals), or a different method of delivery, might result in improvement both in the immunogenicity and the consistency of vaccination for both groups. Further studies will be needed to support this conjecture.

In conclusion, the study of combination DNA vaccines is in its infancy. It is likely that empirical analysis of various combinations of vaccines will be required to gauge their compatibility, and that new methods and models will be needed to truly assess differences in immunogenicity that will arise when vaccines are given in combination. In this study, we make available for the first time, DNA vaccines for RVFV and $\mathrm{CCHFV}$, which can be further analyzed as multiagent vaccines.

\section{Acknowledgments}

The views, opinions and/or findings contained in this report are those of the authors and should not be construed as an official Department of the Army position, policy, or decision unless so designated by other documentation. In conducting research using animals, the investigators adhered to the "Guide for the Care and Use of Laboratory Animals," prepared by the Committee on Care and Use of Laboratory Animals of the Institute of Laboratory Animal Resources, National Research Council (NIH publication No. 86-23, revised 1996). Part of these studies were conducted while Anita McElroy was a National Research Council Postdoctoral Research Associate.

\section{References}

[1] Riemenschneider J, Garrison A, Geisbert J, Jahrling P, Hevey M, Negley D, et al. Comparison of individual and combination DNA vaccines for B. anthracis, Ebola virus, Marburg virus and Venezuelan equine encephalitis virus. Vaccine 2003;21(25-26):4071-80.

[2] Schmaljohn C, Hjelle B. Hantaviruses: a global disease problem. Emerg Infect Dis 1997;3(2):95-104.

[3] Hooper JW, Li D. Vaccines against hantaviruses. Curr Top Microbiol Immunol 2001;256:171-91.

[4] Gonzalez-Scarano F, Nathanson N. Bunyaviruses. Virology, vol. 1. New York: Raven Press; 1996. p. 1473-504.

[5] Ahmad K. More deaths from Rift Valley fever in Saudi Arabia and Yemen. Lancet 2000;356(9239):1422.

[6] Gerdes GH. Rift valley fever. Vet Clin North Am Food Anim Pract 2002;18(3):549-55.

[7] Meadors III GF, Gibbs PH, Peters CJ. Evaluation of a new Rift Valley fever vaccine: safety and immunogenicity trials. Vaccine 1986;4(3):179-84.

[8] Pittman PR, Makuch RS, Mangiafico JA, Cannon TL, Gibbs PH, Peters CJ. Long-term duration of detectable neutralizing antibodies after administration of live-attenuated VEE vaccine and following booster vaccination with inactivated VEE vaccine. Vaccine 1996;14(4):337-43.

[9] Caplen H, Peters C, Bishop D. Mutagen-directed attenuation of Rift Valley fever virus as a method for vaccine development. J Gen Virol 1985;66:2271-7.

[10] Morrill JC, Carpenter L, Taylor D, Ramsburg HH, Quance J, Peters CJ. Further evaluation of a mutagen-attenuated Rift Valley fever vaccine in sheep. Vaccine 1991;9:35-41. 
[11] Morrill JC, Jennings GB, Caplen H, Turell MJ, Johnson AJ, Peters CJ. Pathogenicity and immunogenicity of a mutagen-attenuated Rift Valley fever virus immunogen in pregnant ewes. Am J Vet Res 1987;48(7):1042-7.

[12] Morrill JC, Mebus CA, Peters CJ. Safety and efficacy of a mutagenattenuated Rift Valley fever virus vaccine in cattle. Am J Vet Res 1997;58(10):1104-9.

[13] Niklasson B, Peters CJ, Bengtsson E, Norrby E. Rift valley fever virus vaccine trial: study of neutralizing antibody response in humans. Vaccine 1985;3:123-7.

[14] Burke DS, Monath TP. Flaviviruses. In: Knipe DM, Howley PM, editors. Fields virology, vol. 1. Philadelphia, PA: Lippincott Williams \& Wilkins; 2001. p. 1043-125.

[15] Kunz C, Heinz FX, Hofmann H. Immunogenicity and reactogenicity of a highly purified vaccine against tidk-borne encephalitis. J Med Virol 1980;6:103-9.

[16] Hooper JW, Kamrud KI, Elgh F, Custer D, Schmaljohn CS. DNA vaccination with hantavirus $M$ segment elicits neutralizing antibodies and protects against seoul virus infection. Virology 1999;255(2):269-78.

[17] Hooper JW, Custer DM, Thompson E, Schmaljohn CS. DNA vaccination with the Hantaan virus $M$ gene protects hamsters against three of four HFRS Hantaviruses and elicits a high-titer neutralizing antibody response in rhesus monkeys. J Virol 2001;75(18):8469-77.

[18] Custer DM, Thompson E, Schmaljohn CS, Ksiazek TG, Hooper JW. Active and passive vaccination against hantavirus pulmonary syndrome with Andes virus M genome segment-based DNA vaccine. J Virol 2003;77(18):9894-905.

[19] Schmaljohn C, VanderZanden L, Bray M, Custer D, Meyer B, Li $\mathrm{D}$, et al. Naked DNA vaccines expressing the prM and $\mathrm{E}$ genes of Russian Spring Summer encephalitis virus and Central European encephalitis virus protect mice from homologous and heterologous challenge. J Virol 1997;71:9563-9.

[20] Schmaljohn C, Custer D, VanderZanden L, Spik K, Rossi C, Bray M. Evaluation of tick-borne encephalitis DNA vaccines in monkeys. Virology 1999;263(1):166-74.

[21] Schmaljohn CS, Parker MD, Ennis WH, Dalrymple JM, Collett MS, Suzich JA, et al. Baculovirus expression of the M genome segment of Rift Valley fever virus and examination of antigenic and immunogenic properties of the expressed proteins. Virology 1989;170(1):184-92.

[22] Dalrymple JM, Hasty SE, Kakach LT, Collett MS. Mapping of protective determinants of Rift Valley fever virus using recombinant vaccinia viruses. In: Lerner RA, Ginsberg H, Chanock R, Brown F, editors. Vaccines 89. Cold Spring Harbor, NY: Cold Spring Harbor Laboratory; 1989. p. 371-5.

[23] Besselaar TG, Blackburn NK. Topological mapping of antigenic sites on the Rift Valley fever virus envelope glycoproteins using monoclonal antibodies. Arch Virol 1991;121(1-4):111-24.

[24] Sanchez AJ, Vincent MJ, Nichol ST. Characterization of the glycoproteins of Crimean-Congo hemorrhagic fever virus. J Virol 2002;76(14):7263-75

[25] Vincent MJ, Sanchez AJ, Erickson BR, Basak A, Chretien M, Seidah NG, et al. Crimean-Congo hemorrhagic fever virus glycoprotein proteolytic processing by subtilase SKI-1. J Virol 2003;77(16):8640-9.

[26] Bertolotti-Ciarlet A, Smith J, Strecker K, Paragas J, Altamura LA, McFalls JM, et al. Cellular localization and antigenic characterization of Crimean-Congo hemorrhagic fever virus glycoproteins. J Virol 2005;79(10):6152-61.
[27] Eisenbraun MD, Fuller DH, Haynes JR. Examination of parameters affecting the elicitation of humoral immune responses by particle bombardment-mediated genetic immunization. DNA Cell Biol 1993;12(9):791-7.

[28] Arrington J, Braun RP, Dong L, Fuller DH, Macklin MD, Umlauf $\mathrm{SW}$, et al. Plasmid vectors encoding cholera toxin or the heat-labile enterotoxin from Escherichia coli are strong adjuvants for DNA vaccines. J Virol 2002;76(9):4536-46.

[29] Pertmer TM, Eisenbraun MD, McCabe D, Prayaga SK, Fuller DH, Haynes JR. Gene gun-based nucleic acid immunization: elicitation of humoral and cytotoxic $\mathrm{T}$ lymphocyte responses following epidermal delivery of nanogram quantities of DNA. Vaccine 1995;13(15):1427-30.

[30] Kakach LT, Wasmoen TL, Collett MS. Rift Valley fever virus M segment: use of recombinant vaccinia viruses to study Phlebovirus gene expression. J Virol 1988;62(3):826-33.

[31] Suzich JA, Kakach LT, Collett MS. Expression strategy of a phlebovirus: biogenesis of proteins from the Rift Valley fever virus M segment. J Virol 1990;64(4):1549-55.

[32] Kakach LT, Suzich JA, Collett MS. Rift Valley fever virus M segment: phlebovirus expression strategy and protein glycosylation. Virology 1989;170(2):505-10.

[33] Wasmoen TL, Kakach LT, Collett MS. Rift Valley fever virus M segment: cellular localization of M segment-encoded proteins. Virology 1988;166(1):275-80.

[34] Gerrard SR, Nichol ST. Characterization of the Golgi retention motif of Rift Valley fever virus $\mathrm{G}(\mathrm{N})$ glycoprotein. J Virol 2002;76(23):12200-10

[35] Kim G, McKee Jr KT. Pathogenesis of Hantaan virus infection in suckling mice: clinical virologic, and serologic observations. Am J Trop Med Hyg 1985;34(2):388-95.

[36] McKee Jr KT, Kim GR, Green DE, Peters CJ. Hantaan virus infection in suckling mice: virologic and pathologic correlates. J Med Virol 1985;17(2):107-17.

[37] Ebihara H, Yoshimatsu K, Ogino M, Araki K, Ami Y, Kariwa $\mathrm{H}$, et al. Pathogenicity of Hantaan virus in newborn mice: genetic reassortant study demonstrating that a single amino acid change in glycoprotein G1 is related to virulence. J Virol 2000;74(19):9245-55.

[38] Wichmann D, Grone HJ, Frese M, Pavlovic J, Anheier B, Haller O, et al. Hantaan virus infection causes an acute neurological disease that is fatal in adult laboratory mice. J Virol 2002;76(17):8890-9.

[39] Kamrud KI, Hooper JW, Elgh F, Schmaljohn CS. Comparison of the protective efficacy of naked DNA, DNA-based Sindbis replicon, and packaged Sindbis replicon vectors expressing Hantavirus structural genes in hamsters. Virology 1999;263(1):209-19.

[40] Hooper JW, Custer DM, Schmaljohn CS, Schmaljohn AL. DNA vaccination with vaccinia virus $L 1 R$ and $A 33 R$ genes protects mice against a lethal poxvirus challenge. Virology 2000;266(2):329-39.

[41] Sedegah M, Charoenvit Y, Aguiar J, Sacci J, Hedstrom R, Kumar S, et al. Effect on antibody and T-cell responses of mixing five GMPproduced DNA plasmids and administration with plasmid expressing GM-CSF. Genes Immun 2004;5(7):553-61.

[42] Kong WP, Huang Y, Yang ZY, Chakrabarti BK, Moodie Z, Nabel GJ. Immunogenicity of multiple gene and clade human immunodeficiency virus type 1 DNA vaccines. J Virol 2003;77(23):12764-72.

[43] Musacchio A, Rodriguez EG, Herrera AM, Quintana D, Muzio V. Multivalent DNA-based immunization against hepatitis B virus with plasmids encoding surface and core antigens. Biochem Biophys Res Commun 2001;282(2):442-6. 